Available online on 15.08 .2020 at http://jddtonline.info
Open Access to Pharmaceutical and Medical Research
unrestricted non-commercial use, provided the original work is properly cited

Open $\odot$ Access

Research Article

\title{
Lipid ratios as a predictive marker of Subclinical Atherosclerosis inflammation among Type 1 Diabetic patients with Thyroid Dysfunction
}

\author{
Walid Hassene HAMRI'.2, Mustapha DIAF1*, Noria HARIR1.2, Mohamed HADJ HABIB ${ }^{3}$ \\ 1. Department of Biology, Faculty of Natural and Life Sciences, Djillali Liabes University of Sidi-bel-Abbes, Algeria \\ 2. Laboratory of Molecular Microbiology, Proteomics and Health, Department of Biology, Faculty of Natural and Life Sciences, Djillali Liabes \\ University of Sidi-bel-Abbes, Algeria
}

3. Department of Medecine, Faculty of Medicine, Djillali Liabes University of Sidi-bel-Abbes, Algeria

\begin{abstract}
Background and Objectives: Epidemiological inquiries on the subclinical atherosclerotic disease in type 1 diabetes associated with autoimmune thyroid disease are scarce. Our aim was to evaluate the risk of developing atherosclerosis via lipid ratios by comparing two groups of patients according to their TSH (Thyroid-stimulating hormone) status.

Methods: A retrospective study including 190 patients (13-74 years) with confirmed type 1 diabetes divided into the following groups (patients with serum TSH $<2.5 \mu \mathrm{IU} / \mathrm{mL}$ vs. patients with serum TSH $\geq 2.5 \mu \mathrm{IU} / \mathrm{mL}$ ). Autoimmune thyroid disease was classified according to clinical, biological, and follow up data.

Results: Our study showed a slight predominance of females (50.50\%), with an average age of $29.25 \pm 11.39$ years. The prevalence of hypothyroidism was $14.7 \%$, hyperthyroidism $6.3 \%$, and anti-TPO $16.31 \%$. Significant differences were observed between lipid profiles such as HDL and TG ( $p=0.008 ; p=0.04$, respectively). The results showed that the $3^{\text {rd }}(\mathrm{OR}=2.28[0.72-7.20] ; \mathrm{p}=0.15)$ and the fourth quartiles $(\mathrm{OR}=1.9$ [0.61-5.83]; $p=0.26$ ) of TC/HDL ratio were higher in patients with serum $\mathrm{TSH} \geq 2.5 \mu \mathrm{IU} / \mathrm{mL}$ group. Similarly, we noticed higher concordant values on the last quartile $\left(4^{\text {th }}\right)$ of LDL/HDL ratio with $\mathrm{p}$ values of 0.06 as well as the two quartiles ( $\left.2^{\text {ndand }} 4^{\text {th }}\right)$ of TG/HDL ratio with $\mathrm{p}$ values of 0.03 and 0.04 , respectively. In both groups, lipid ratios were slightly higher in males compared to females' patients.
\end{abstract}

Conclusion: The risk of atherosclerosis was higher in patients with elevated TSH concentrations. Therefore, early detection of thyroid dysfunction and associated dyslipidemia is essential for effective prevention of premature cardiovascular morbidity and mortality.

Keywords: Subclinical atherosclerotic disease, autoimmune thyroid disease, TSH, type 1 diabetes

Article Info: Received 09 June 2020; Review Completed 17 July 2020; Accepted 24 July 2020; Available online 15 August 2020

Cite this article as: Hamri WH, Diaf M, Harir N, Hadj Habib M, Lipid ratios as a predictive marker of Subclinical Atherosclerosis inflammation among Type 1 Diabetic patients with Thyroid Dysfunction, Journal of Drug Delivery and Therapeutics. 2020; 10(4-s):112-129 http://dx.doi.org/10.22270/jddt.v10i4-s.4307

*Address for Correspondence: Mustapha DIAF, Department of Biology, Faculty of Natural and Life Sciences, Djillali Liabes University of Sidi-bel-Abbes, Algeria

\section{INTRODUCTION}

Approximately 4.2 million persons aged between 20-79 years are estimated to die as a result of diabetes and its complications in 2019 1. In the same year, the International Diabetes Federation (IDF) estimated that 463 million people around the world aged 20-79 years had diabetes, and by 2035 this number is expected to rise to 587.4 million 1 .

Type 1 diabetes (T1D) is an auto-immune disease leading to absolute insulin deficiency 2 , with prevalence estimated to be 207 cases per 100,000 equivalents to 1 T1D for 482 children under $15^{3}$. It can be related to other auto-immune disorders that may affect the control of diabetes by disrupting the function of some organs ${ }^{2}$. Commonly known, this disease is closely associated with autoimmune thyroid disease (AITD) in clinical practice because these endocrinopathies are linked by the same pathophysiological mechanism 4,5. They share an autoimmune predisposition, and some genetic factors might contribute to the coexistence of AITD and T1D 6 , and indeed up to a third of patients with T1D ultimately develop thyroid dysfunction at an early age compared with the general population 7 .

It has been also reported that AITD occurs in $17 \%$ to $30 \%$ of individuals with T1D, and these patients are at higher risk of both autoimmune-induced hyperthyroidism (Graves's disease) as well as hypothyroidism (Hashimoto's thyroiditis) 8. Thyroid autoantibodies can be detected at the initial diagnosis or may be detected over time, after diagnosis 2 . The prevalence of positive antithyroid peroxidase antibodies (anti-TPO) is estimated to be ranged between 2\% and 10\% in the general population, whereas in the population of T1D patients it is much higher, reaching the level from $15 \%$ to $30 \%{ }^{9}$.

According to some of the recognized associations, global guidelines currently recommend routine screening for 
thyroid function and anti-TPO in patients with T1D. Specifically, the American Diabetes Association (ADA) guideline document advises screening for anti-TPO and thyroid function soon after diagnosis and then to consider rechecking thyroid function every two years ${ }^{10}$.

Undiagnosed thyroid disorder may impair metabolic control in patients with T1D including the synthesis, mobilization, and catabolism of lipids which may amplify the risk of atherosclerosis disease 11,12. Diabetes mellitus, in particular T1D, which was highly associated with lipid abnormalities 13 , was also known to dramatically increasing the risk of atherosclerotic cardiovascular disease especially to those at a younger age due to their long duration of diabetes 14 .

To that end, we aimed in this scientific study to assess and to compare the prevalence of known and undiagnosed thyroid disease and to analyze the thyroid autoantibody profile among patients with T1D in order to investigate the potential atherosclerosis risk, which has greater predictive power for a cardiovascular complication, by evaluating blood lipid ratios in Algerian type 1 diabetic patients since no similar studies have been performed before.

\section{PATIENTS AND METHODS}

Our transverse retrospective observational study was performed using a database of 190 patients $\geq 13$ years with a confirmed T1D according to the WHO criteria 15 who had been referred to the University Hospital Center in Sidi-BelAbbes, northwestern Algeria in the years 2009 to 2019 and remained under the care of the Endocrinology Department of the hospital thereafter. Patients' medical records were reviewed for medical history, other associated diseases, biochemical parameters, and complications of the diabetic disease. All patients with previously diagnosed with T1D, aged more than 13 years with no history of any cardiovascular disease were involved in the analysis. Though, patients with T1D aged less than 13 years, missing the medical records of the disease, and missing informed consent were excluded. Ethical approval for the study was granted by the Ethics Committee of the University Hospital Center in which the study was carried out.

For all patients, anthropometric parameters (body weight and height, body mass index "BMI" and waist circumference) were available on the patient's medical record. Blood pressure was measured with a sphygmomanometer in a supine position followed by a second measurement (after a few minutes) in a standing position. Hypertension was defined by blood pressure (BP) in the spine position of $140 / 90 \mathrm{mmHg}$ or more or by current anti-hypertensive treatment. The latest biochemical assessment including fasting blood glucose, glycated hemoglobin (HbA1c), highsensitivity C-reactive protein (hs-CRP) $(<6 \mathrm{mg} / \mathrm{L}$ ), urea (0.15-0.45 g/L), serum creatinine $(4-10 \mathrm{mg} / \mathrm{L})$ and lipid parameters, namely; total cholesterol (TC) (1.5-2 g/L), highdensity lipoprotein cholesterol (HDL) $(\geq 0.45 \mathrm{~g} / \mathrm{L})$, lowdensity lipoprotein cholesterol (LDL) $(0.5-1.5 \mathrm{~g} / \mathrm{L})$ and triglycerides (TG) (0.5-1.5 g/L), microalbuminuria $(>30$ $\mathrm{mg} / 24 \mathrm{~h}$ ), thyroid-stimulating hormone (TSH) $0.27-4.25$ $\mu \mathrm{IU} / \mathrm{mL}$ ), Anti-thyroid peroxidase antibodies (Anti-TPO) (< $30 \mathrm{IU} / \mathrm{mL}$ ) were taken from patients' medical records. AntiTPO was defined as positive $(>30 \mathrm{IU} / \mathrm{mL})$ in at least one sample obtained within the period of hospitalization. Moreover, lipid ratios as indicators of atherogenic risk were calculated; TC/HDL, LDL/HDL, and TG/HDL.

\section{Statistical analysis}

Concerning the statistical analytical study, the data were summarized using mean and standard deviation values with its respective $95 \%$ confidence intervals (95\% CI) for continuous variables and percentages (\%) and relative frequencies for qualitative variables.

Chi-square test and Student's $t$-test were used to compare qualitative and quantitative variables between the two groups. Significant differences were established when the $p$ value is less than or equal to $0.05(p \leq 0.05)$.

To explore the differences of categorical variables such as serum lipid ratios and to assess their clinical utility to identify the atherosclerosis risk in patients with T1D according to their TSH status multivariate logistic regression was applied. All data were computed and analyzed using SPSS software (SPSS 22, IBM Corporation; Chicago, IL. August 2013).

\section{RESULTS}

We identified 190 type 1 diabetic patients (49.50\% males and $50.50 \%$ females) with a male-to-female ratio of 0.98 . Among all the involved patients, 73 (38.4\%) patients presented with $\mathrm{TSH}<2.5 \mu \mathrm{IU} / \mathrm{mL}$ while $117(61.6 \%)$ patients with $\mathrm{TSH} \geq 2.5 \mu \mathrm{IU} / \mathrm{mL}$.The mean age of the sample was $29.25 \pm 11.39$ years (range 13-74) and mean duration of diabetes was $12.20 \pm 8.45$ years. Meanwhile, the mean age of patients with $\mathrm{TSH}<2.5 \mu \mathrm{IU} / \mathrm{mL}$ was significantly lower than that of patients with $\mathrm{TSH} \geq 2.5 \mu \mathrm{IU} / \mathrm{mL}(25.60 \pm 10.11$ years vs. $31.52 \pm 11.59$ years, $p<10^{-3}$ ) (Table 2). The most affected age group was the "20-29 years" with a rate of $(38.40 \%)$, followed by the "30-39 years" group with (22.60\%). However, the least affected age group was the " $\geq 60$ years". The overall prevalence of underweight, normal weight, overweight, and obesity was (22.10\%), (65.30\%), (11.10\%), and $(1.50 \%)$, respectively (Table 1$)$.

In the current study, significant differences were found concerning the well-known complications of diabetes. The differences were most pronounced for diabetic nephropathy, diabetic retinopathy, hypothyroidism, hyperthyroidism and hypertension which all differed significantly between the two groups $\left(16.30 \%, p=<10^{-3} ; 21.00 \%, p=0.04 ; 14.70 \%\right.$, $p=<10^{-3} ; 6.30 \%, p=<10^{-3}$; and $15.80 \%, p=0.02$; respectively) (Table 1).

Concerning the Laboratory data for both groups, there were no significant differences between them in terms of age at first diagnosis, anthropometric measurement (body weight, height, and waist circumference), fasting plasma glucose, HbA1c level, Total cholesterol (TC), LDL-c, TC/HDL-c ratio, LDL/HDL-c ratio (all $p>0.05$ ) (Table 2). However, significantly higher systolic blood pressure (SBP) $(p=0.001)$ and diastolic blood pressure (DBP) $(p=0.01)$ was found in TSH $\geq 2.5 \mu \mathrm{IU} / \mathrm{mL}$ compared to $\mathrm{TSH}<2.5 \mu \mathrm{IU} / \mathrm{mL}$ T1D patients, on the other hand, high-sensitivity C-Reactive Protein (hs-CRP) was lower in subjects with serum TSH < $2.5 \mu \mathrm{IU} / \mathrm{mL}$ than that with serum $\mathrm{TSH} \geq 2.5 \mu \mathrm{IU} / \mathrm{mL}$ $(6.61 \pm 14.02 \mathrm{mg} / \mathrm{L} \quad$ vs. $36.45 \pm 58.21 \mathrm{mg} / \mathrm{L}, \quad p=0.001$; respectively). With regard to lipid levels, as shown in (Table 2 ) when the t-test was applied to the HDL-c and triglycerides for both groups, $p$-value was $0.008,0.04$ respectively which was statistically significant. Further, it was found that TG/HDL-c ratio was significantly higher in the group with serum TSH $\geq 2.5 \mu \mathrm{IU} / \mathrm{mL}$ ( $p=0.03$ ) (Table 2 ).

With respect to renal function, patients with serum TSH $\geq$ $2.5 \mu \mathrm{IU} / \mathrm{mL}$ had increased plasma levels of creatinine and urea and microalbuminuria $(17.07 \pm 22.12 \mathrm{~g} / \mathrm{L}, \quad p=0.002$; $0.53 \pm 0.48 \mathrm{~g} / \mathrm{L}, \quad p=<10^{-3} ;$ and $218.42 \pm 553.14 \mathrm{mg} / 24 \mathrm{~h}$, $p=0.05$; respectively).

Regarding patients who had both T1D and thyroid dysfunction, Anti-TPO antibodies were higher in patients 
with serum $\mathrm{TSH} \geq 2.5 \mu \mathrm{IU} / \mathrm{mL}$ than that with serum $\mathrm{TSH}<$ $2.5 \mu \mathrm{IU} / \mathrm{mL}(99.42 \pm 123.66 \mathrm{IU} / \mathrm{mL}$ vs. $7.99 \pm 10.04 \mathrm{IU} / \mathrm{mL}$, respectively, $p=0.006$ ).

For the whole study population, the multivariate regression between lipid ratios quartiles, as powerful predictors of atherosclerosis, plotted that the $3^{\text {rd }}(\mathrm{OR}=2.28$ [0.72-7.20]; $p=0.15)$ and the fourth quartiles $(\mathrm{OR}=1.9$ [0.61-5.83]; $p=0.26$ ) of TC/HDL ratio were higher in both groups as described in (Table 3). Furthermore, we noticed higher disconcordant values in the $4^{\text {th }}$ quartile of the LDL/HDL ratio with $p$-value 0.06 . Likewise, there were a higher significant differences in the $\left(2^{\text {ndand }} 4^{\text {th }}\right)$ quartiles of the TG/HDL ratio disclosed an Odd ratio of 0.26 [0.07-0.89] $(p=0.03)$ and $(\mathrm{OR}=$ 0.28 [0.08-0.97]; $p=0.04$ ), respectively.
As shown in Figure 1, in males and females, when comparing lipid ratios between the two groups, TC/HDL, LDL/HDL, and TG/HDL ratios were slightly higher in males compared to females' type 1 diabetic patients. In addition, higher TC, LDL, and TG levels were observed in males compared to females with serum TSH $<2.5 \mu \mathrm{IU} / \mathrm{mL}$. Meanwhile, females with serum $\mathrm{TSH} \geq 2.5 \mu \mathrm{IU} / \mathrm{mL}$ had high levels of TC and LDL compared to male patients. Similarly, TG levels were higher in males compared to females with serum TSH $\geq 2.5 \mu \mathrm{IU} / \mathrm{mL}$.

Concerning the subgroups divided by the TSH cut-off level of $2.5 \mu \mathrm{IU} / \mathrm{mL}$ (Figure 2). There was a higher prevalence of anti-TPO in patients with TSH $\geq 2.5 \mu \mathrm{IU} / \mathrm{mL}$ [64.44\% vs. $35.56 \%$ ). Likewise, we did observe a statistically significant difference in the prevalence of anti-TPO among patients with serum TSH below $2.5 \mu \mathrm{IU} / \mathrm{mL}$ [93.33\% vs. $6.67 \%$ ]).

Table 1 :Basic characteristics of the participants

\begin{tabular}{|c|c|c|c|c|}
\hline Variables & $\begin{array}{c}\text { All Patients } \\
\text { n=190 } \\
\text { Number (\%) }\end{array}$ & $\begin{array}{c}\text { TSH }<2.5(\mu \mathrm{IU} / \mathrm{mL}) \\
\mathrm{n}=73 \\
\text { Number }(\%)\end{array}$ & $\begin{array}{c}\text { TSH } \geq 2.5(\mu \mathrm{IU} / \mathrm{mL}) \\
\text { n=117 } \\
\text { Number }(\%)\end{array}$ & $p$-value \\
\hline \multicolumn{5}{|l|}{ Gender, $\mathrm{n}(\%)$} \\
\hline Male & $94(49.50)$ & $30(41.10)$ & $64(54.70)$ & \multirow[t]{2}{*}{0.069} \\
\hline Female & $96(50.50)$ & $43(58.90)$ & $53(45.30)$ & \\
\hline \multicolumn{5}{|l|}{ Age groups, years } \\
\hline [13-19] & $38(20)$ & $24(32.90)$ & $14(12.00)$ & \multirow{6}{*}{$<10^{-3 *}$} \\
\hline [20-29] & $73(38.40)$ & $25(34.30)$ & $48(41.00)$ & \\
\hline [30-39] & $43(22.60)$ & $16(21.90)$ & $27(23.10)$ & \\
\hline$[40-49]$ & $22(11.60)$ & $6(8.20)$ & $16(13.70)$ & \\
\hline [50-59] & $12(6.30)$ & $2(2.70)$ & $10(8.50)$ & \\
\hline$\geq 60$ & $2(1.10)$ & $0(0.00)$ & $2(1.70)$ & \\
\hline \multicolumn{5}{|l|}{ Smoking history, n (\%) } \\
\hline Male & $23(21.10)$ & $7(9.60)$ & $16(13.70)$ & 0.40 \\
\hline Female & -.- & -- & -- & -- \\
\hline \multicolumn{5}{|l|}{ Prevalence of weight categories, $\mathrm{n}(\%)$} \\
\hline Underweight, $\mathrm{BMI}<18.5 \mathrm{Kg} / \mathrm{m}^{2}$ & $42(22.10)$ & $19(26.00)$ & $23(19.70)$ & \multirow{4}{*}{0.45} \\
\hline Normal weight, $B M I=18.5-25.0 \mathrm{Kg} / \mathrm{m}^{2}$ & $124(65.30)$ & $45(61.60)$ & $79(67.50)$ & \\
\hline Overweight, $\mathrm{BMI}=25.0-29.9 \mathrm{Kg} / \mathrm{m}^{2}$ & $21(11.10)$ & $8(11.00)$ & $13(11.10)$ & \\
\hline Obesity, BMI $\geq 30 \mathrm{Kg} / \mathrm{m}^{2}$ & $3(1.50)$ & $1(1.40)$ & $2(1.70)$ & \\
\hline \multicolumn{5}{|l|}{ Other associated diseases, $n(\%)$} \\
\hline Low visual acuity & $63(33.20)$ & $14(19.20)$ & $49(41.90)$ & $<10^{-3 *}$ \\
\hline Diabetic retinopathy & $40(21.00)$ & $11(15.10)$ & $29(24.80)$ & $0.04 *$ \\
\hline Diabetic nephropathy & $31(16.30)$ & $1(1.40)$ & $30(25.70)$ & $<10^{-3 *}$ \\
\hline Hypertension & $30(15.80)$ & $6(8.20)$ & $24(20.50)$ & $0.02 *$ \\
\hline Hypothyroidism & $28(14.70)$ & $0(0.00)$ & $28(23.90)$ & $<10^{-3 *}$ \\
\hline Hyperthyroidism & $6(6.30)$ & $12(16.40)$ & $0(0.00)$ & $<10^{-3 *}$ \\
\hline Anemia & $60(31.60)$ & $22(30.10)$ & $38(32.50)$ & 0.73 \\
\hline Dyslipidemia & $7(3.70)$ & $2(2.70)$ & $5(4.30)$ & 0.58 \\
\hline \multicolumn{5}{|l|}{ Symptoms and signs, n (\%) } \\
\hline \multicolumn{5}{|l|}{ Cardinal syndrome, $\mathrm{n}(\%)$} \\
\hline Weight loss & $81(42.60)$ & $30(41.10)$ & $51(43.60)$ & 0.73 \\
\hline Polyuria-Polydipsia & $171(90.00)$ & $65(89.00)$ & $106(90.60)$ & 0.54 \\
\hline Asthenia & $54(28.40)$ & $15(20.50)$ & $39(33.30)$ & $0.05^{*}$ \\
\hline Overeating & $107(56.30)$ & $32(43.80)$ & $75(64.10)$ & $0.03^{*}$ \\
\hline \multicolumn{5}{|l|}{ Medical care, $n(\%)$} \\
\hline Diabetic foot & $29(15.30)$ & $5(6.80)$ & $24(20.50)$ & $<10^{-3 *}$ \\
\hline Ketosis on inaugural diabetes & $25(13.20)$ & $13(17.80)$ & $12(10.30)$ & 0.09 \\
\hline Ketosis on diabetes & $41(21.60)$ & $14(19.20)$ & $27(23.10)$ & 0.64 \\
\hline Severe hyperglycemia & $14(7.40)$ & $6(8.20)$ & $8(6.80)$ & 0.58 \\
\hline Severe hypoglycemia & $6(3.20)$ & $3(4.10)$ & $3(2.60)$ & 0.50 \\
\hline \multicolumn{5}{|l|}{ Family history, n (\%) } \\
\hline Hypertension & $32(16.80)$ & $11(15.10)$ & $21(17.90)$ & 0.60 \\
\hline Type 1 diabetes & $22(11.60)$ & $8(11.00)$ & $14(12.00)$ & 0.73 \\
\hline Type 2 diabetes & $79(41.60)$ & $25(34.20)$ & $54(46.20)$ & 0.10 \\
\hline Goiter & $8(4.20)$ & $5(6.80)$ & $3(2.60)$ & 0.15 \\
\hline
\end{tabular}

$\left({ }^{*}\right)$ percentages were compared with Chi-square test, $p \leq 0.05$ was considered as significant; BMI: body mass index. 
Table 2: Comparison of clinical characteristics between patients' serum TSH levels subgroups

\begin{tabular}{|c|c|c|c|c|c|c|c|}
\hline \multirow[t]{2}{*}{ Variables } & \multicolumn{2}{|c|}{ All Patients, n=351 } & \multicolumn{2}{|c|}{$\begin{array}{c}\text { TSH }<2.5(\mu \mathrm{IU} / \mathrm{mL}), \\
n=155\end{array}$} & \multicolumn{2}{|c|}{$\begin{array}{c}\mathrm{TSH} \geq 2.5(\mu \mathrm{IU} / \mathrm{mL}), \\
\mathrm{n}=196\end{array}$} & \multirow[t]{2}{*}{$\begin{array}{c}p- \\
\text { value }\end{array}$} \\
\hline & Mean \pm SD & $95 \% \mathrm{CI}$ & Mean \pm SD & $95 \% \mathrm{CI}$ & Mean \pm SD & 95\%CI & \\
\hline Mean age (years) & $29.25 \pm 11.39$ & $\begin{array}{l}27.62- \\
30.88\end{array}$ & $\begin{array}{c}25.60 \pm \\
10.11\end{array}$ & $\begin{array}{l}23.24- \\
27.96\end{array}$ & $\begin{array}{c}31.52 \pm \\
11.59\end{array}$ & $\begin{array}{l}29.40- \\
33.64\end{array}$ & $<10^{-3} *$ \\
\hline $\begin{array}{l}\text { Diabetes duration } \\
\text { (years) }\end{array}$ & $12.20 \pm 8.45$ & $\begin{array}{l}10.99- \\
13.41\end{array}$ & $9.48 \pm 8.01$ & $\begin{array}{l}7.61- \\
11.35\end{array}$ & $13.90 \pm 8.30$ & $\begin{array}{l}12.38- \\
15.42\end{array}$ & $<10^{-3} *$ \\
\hline $\begin{array}{l}\text { Age at } 1^{\text {st }} \text { diagnosis } \\
\text { (years) }\end{array}$ & $17.07 \pm 9.04$ & $\begin{array}{l}15.77- \\
18.36\end{array}$ & $16.15 \pm 9.16$ & $\begin{array}{l}14.01- \\
18.29\end{array}$ & $17.64 \pm 8.95$ & $\begin{array}{l}16.00- \\
19.28\end{array}$ & 0.27 \\
\hline Body height (m) & $1.66 \pm 0.07$ & $1.65-1.68$ & $1.65 \pm 0.07$ & $1.64-1.67$ & $1.67 \pm 0.08$ & $1.66-1.69$ & 0.12 \\
\hline Body weight (Kg) & $58.63 \pm 11.67$ & $\begin{array}{l}56.85- \\
60.40\end{array}$ & $\begin{array}{c}57.01 \pm \\
11.71\end{array}$ & $\begin{array}{l}54.16- \\
59.87\end{array}$ & $\begin{array}{c}59.68 \pm \\
11.58\end{array}$ & $\begin{array}{l}57.41- \\
61.96\end{array}$ & 0.14 \\
\hline $\mathrm{BMI}\left(\mathrm{Kg} / \mathrm{m}^{2}\right)$ & $21.00 \pm 3.55$ & $\begin{array}{l}20.46- \\
21.54\end{array}$ & $20.82 \pm 3.65$ & $\begin{array}{l}19.93- \\
21.71\end{array}$ & $21.12 \pm 3.50$ & $\begin{array}{l}20.43- \\
21.81\end{array}$ & 0.59 \\
\hline $\begin{array}{l}\text { Waist circumference } \\
(\mathrm{cm})\end{array}$ & $81.72 \pm 10.41$ & $\begin{array}{l}78.88- \\
84.56\end{array}$ & $78.63 \pm 6.55$ & $\begin{array}{l}75.13- \\
82.12\end{array}$ & $\begin{array}{c}83.03 \pm \\
11.48\end{array}$ & $\begin{array}{l}79.25- \\
86.80\end{array}$ & 0.15 \\
\hline SBP (mmHg) & $113.1 \pm 13.4$ & $\begin{array}{l}111.2- \\
115.0\end{array}$ & $109.2 \pm 10.7$ & $\begin{array}{l}106.7- \\
111.7\end{array}$ & $115.6 \pm 14.3$ & $\begin{array}{l}113.0- \\
118.2\end{array}$ & $0.001^{*}$ \\
\hline DBP (mmHg) & $65.7 \pm 9.3$ & $64.4-67.1$ & $63.6 \pm 9.1$ & $61.4-65.7$ & $67.1 \pm 9.2$ & $65.4-68.8$ & $0.01 *$ \\
\hline $\begin{array}{l}\text { Fasting plasma glucose } \\
(\mathrm{g} / \mathrm{L})\end{array}$ & $2.92 \pm 1.32$ & $2.73-3.11$ & $2.77 \pm 1.21$ & $2.48-3.05$ & $3.02 \pm 1.38$ & $2.76-3.27$ & 0.20 \\
\hline HbA1c (\%) & $9.69 \pm 2.21$ & $9.34-10.04$ & $9.31 \pm 2.15$ & $8.75-9.87$ & $9.93 \pm 2.22$ & $9.47-10.38$ & 0.09 \\
\hline hs CRP (mg/L) & $18.55 \pm 40.76$ & $9.17-27.93$ & $6.61 \pm 14.02$ & $\begin{array}{l}2.40- \\
10.82\end{array}$ & $\begin{array}{c}36.45 \pm \\
58.21\end{array}$ & $\begin{array}{l}14.71- \\
58.19\end{array}$ & $0.001^{*}$ \\
\hline Total cholesterol (g/L) & $1.60 \pm 0.35$ & $1.54-1.66$ & $1.61 \pm 0.32$ & $1.52-1.70$ & $1.59 \pm 0.37$ & $1.51-1.67$ & 0.77 \\
\hline HDL-c (g/L) & $0.43 \pm 0.12$ & $0.41-0.46$ & $0.47 \pm 0.12$ & $0.44-0.51$ & $0.41 \pm 0.11$ & $0.38-0.44$ & $0.008^{*}$ \\
\hline LDL-c (g/L) & $0.95 \pm 0.28$ & $0.89-1.00$ & $0.98 \pm 0.26$ & $0.90-1.07$ & $0.92 \pm 0.30$ & $0.85-1.00$ & 0.31 \\
\hline Triglycerides (g/L) & $0.92 \pm 0.62$ & $0.82-1.02$ & $0.79 \pm 0.45$ & $0.67-0.91$ & $1.00 \pm 0.70$ & $0.85-1.15$ & $0.04 *$ \\
\hline TC/HDL-c & $3.83 \pm 1.14$ & $3.61-4.04$ & $3.61 \pm 0.91$ & $3.30-3.91$ & $3.95 \pm 1.23$ & $3.65-4.24$ & 0.14 \\
\hline LDL/HDL-c & $2.30 \pm 0.85$ & $2.14-2.46$ & $2.16 \pm 0.75$ & $1.91-2.40$ & $2.38 \pm 0.89$ & $2.16-2.60$ & 0.19 \\
\hline TG/HDL-c & $2.45 \pm 2.25$ & $2.02-2.88$ & $1.93 \pm 1.36$ & $1.49-2.38$ & $2.73 \pm 2.57$ & $2.12-3.35$ & $0.03^{*}$ \\
\hline Creatinine (mg/L) & $13.62 \pm 18.48$ & $\begin{array}{l}10.76- \\
16.48\end{array}$ & $7.69 \pm 5.93$ & $6.16-9.23$ & $\begin{array}{c}17.07 \pm \\
22.12\end{array}$ & $\begin{array}{l}12.75- \\
21.40\end{array}$ & $0.002^{*}$ \\
\hline Urea (g/L) & $0.42 \pm 0.41$ & $0.36-0.49$ & $0.24 \pm 0.10$ & $0.22-0.27$ & $0.53 \pm 0.48$ & $0.44-0.63$ & $<10^{-3 *}$ \\
\hline $\begin{array}{l}\text { Microalbuminuria } \\
(\mathrm{mg} / 24 \mathrm{~h})\end{array}$ & $\begin{array}{c}146.55 \pm \\
436.96\end{array}$ & $\begin{array}{l}54.51- \\
238.60\end{array}$ & $40.75-78.50$ & $\begin{array}{l}14.19- \\
67.32\end{array}$ & $\begin{array}{l}218.42- \\
553.14\end{array}$ & $\begin{array}{l}65.95- \\
370.88\end{array}$ & $0.05^{*}$ \\
\hline $\mathrm{TSH}(\mu \mathrm{IU} / \mathrm{mL})$ & $4.79 \pm 9.45$ & $3.44-6.15$ & $1.65 \pm 0.79$ & $1.46-1.83$ & $6.76 \pm 11.62$ & $4.63-8.89$ & $<10^{-3 *}$ \\
\hline Anti-TPO (IU/mL) & $\begin{array}{l}76.57 \pm \\
114.11\end{array}$ & $\begin{array}{l}47-09- \\
106.05\end{array}$ & $7.99 \pm 10.04$ & $\begin{array}{l}2.43- \\
13.56\end{array}$ & $\begin{array}{l}99.42 \pm \\
123.66\end{array}$ & $\begin{array}{l}62.77- \\
136.58\end{array}$ & $0.006^{*}$ \\
\hline
\end{tabular}

$\left(^{*}\right)$ means were compared with independent sample Student's $t$-test, a $p<0.05$ was considered as significant; SD: standard deviation; CI: confidence interval; HbA1c: glycosylated hemoglobin; BMI: body mass index; SBP: systolic blood pressure; DBP: diastolic blood pressure; hs-CRP: high-sensitivity C-reactive protein; TC: total cholesterol; HDL-c: high-density lipoprotein cholesterol; LDL-c: low-density lipoprotein cholesterol; TG: triglycerides; TSH: thyroid-stimulating hormone; Anti-TPO: antithyroperoxidase antibodies. 
Table 3: Crude "Odds Ratio" of blood lipid ratios quartiles associated with serum TSH levels

\begin{tabular}{|c|c|c|c|c|}
\hline Variables & $\begin{array}{c}\text { TSH }<(2.5 \mu I U / m L) \\
n=73 \\
\text { Number }(\%)\end{array}$ & $\begin{array}{c}\text { TSH } \geq 2.5(\mu I U / m L) \\
n=117 \\
\text { Number }(\%)\end{array}$ & Odds ratio (95\% CI OR) & $\begin{array}{c}p- \\
\text { value }\end{array}$ \\
\hline \multicolumn{5}{|l|}{ TC/HDL ratio } \\
\hline $1^{\text {st }}$ quartile (2.09-3.00) & $12(31.6)$ & $15(21.4)$ & Reference & --- \\
\hline $2^{\text {nd }}$ quartile (3.01-3.63) & $11(28.9)$ & $16(22.9)$ & $1.61[0.39-3.42]$ & 0.78 \\
\hline $3^{\text {rd }}$ quartile (3.64-4.34) & 7 (18.4) & $20(28.6)$ & $2.28[0.72-7.20]$ & 0.15 \\
\hline $4^{\text {th }}$ quartile $(4.35-8.10)$ & $8(21.1)$ & $19(27.1)$ & $1.90[0.61-5.83]$ & 0.26 \\
\hline \multicolumn{5}{|l|}{ LDL/HDL ratio } \\
\hline $1^{\text {st }}$ quartile $(0.73-1.74)$ & $13(34.2)$ & $14(20.3)$ & Reference & --- \\
\hline $2^{\text {nd }}$ quartile (1.85-2.13) & $10(26.3)$ & $16(23.2)$ & $1.48[0.49-4.43]$ & 0.47 \\
\hline $3^{\text {rd }}$ quartile (2.14-2.74) & 9 (23.7) & $19(27.5)$ & $1.96[0.65-5.85]$ & 0.22 \\
\hline $4^{\text {th }}$ quartile $(2.75-5.14)$ & $6(15.8)$ & $20(29)$ & $3.09[0.94-10.11]$ & 0.06 \\
\hline \multicolumn{5}{|l|}{ TG/HDL ratio } \\
\hline $1^{\text {st }}$ quartile $(0.45-1.27)$ & $13(34.2)$ & $15(21.4)$ & Reference & --- \\
\hline $2^{\text {nd }}$ quartile (1.28-1.85) & $8(21.1)$ & $18(25.7)$ & $0.26[0.07-0.89]$ & $0.03 *$ \\
\hline $3^{\text {rd }}$ quartile (1.86-2.63) & $12(31.6)$ & $15(21.4)$ & $0.51[0.14-1.83]$ & 0.30 \\
\hline $4^{\text {th }}$ quartile (2.64-14.95) & $5(13.2)$ & $22(31.4)$ & $0.28[0.08-0.97]$ & $0.04 *$ \\
\hline
\end{tabular}

$\left({ }^{*}\right)$ multivariate logistic regression significant at $p=0.05$; CI, confidence interval; OR, Odd ratio; Q, quartiles; TSH: thyroidstimulating hormone $(\mu \mathrm{IU} / \mathrm{mL} / \mathrm{L})$; TC: total cholesterol (g/L); LDL: low-density lipoprotein cholesterol (g/L); HDL: highdensity lipoprotein cholesterol (g/L); TG: triglycerides (g/L)

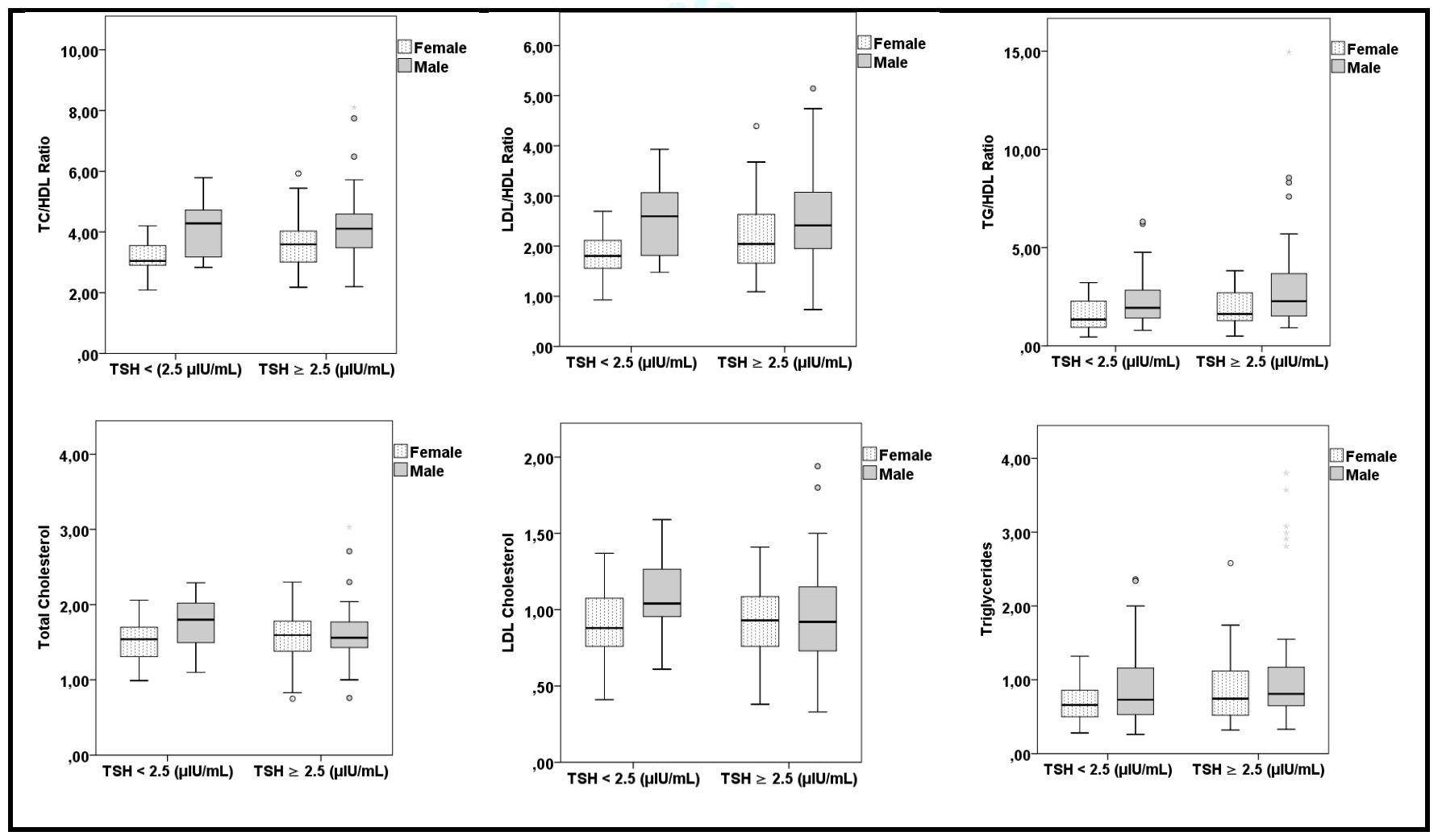

Figure 1: Comparison of lipid ratios levels between patients with serum TSH $<2.5(\mu \mathrm{IU} / \mathrm{mL})$ and with serum TSH $\geq 2.5$ $(\mu \mathrm{IU} / \mathrm{mL})$ according to the patients' gender 


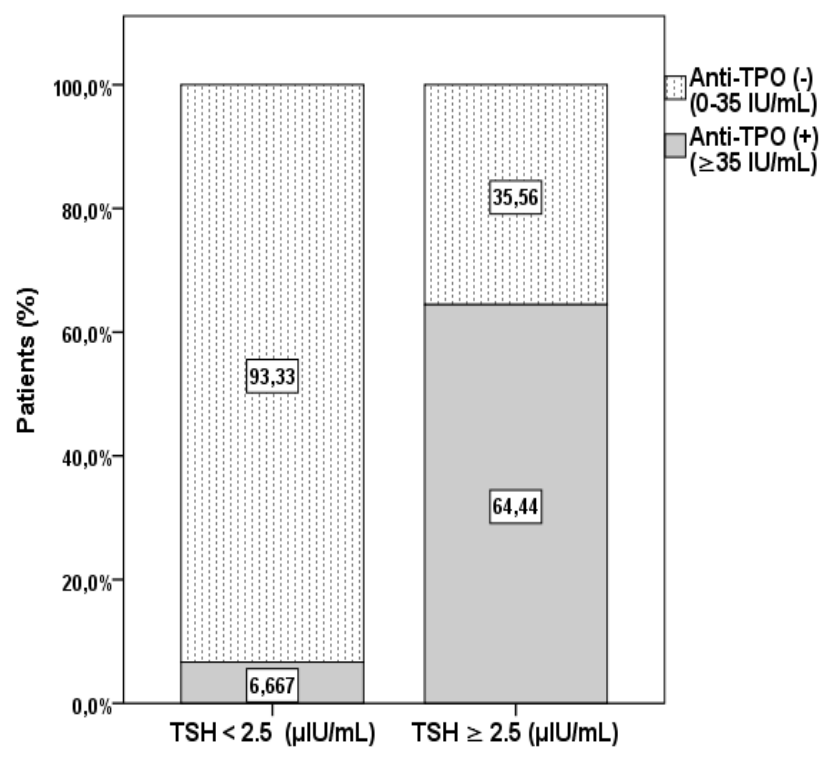

Figure 2: Prevalence of Anti-TPO according to serum TSH concentrations

\section{DISCUSSION}

The objective of the present study was to demonstrate, in patients with T1D, through strong predictors like lipids and their related ratios the risk of developing atherosclerosis by comparing two groups of patients according to their TSH status (patients with TSH $<2.5 \mu \mathrm{IU} / \mathrm{mL} v$ s. patients with TSH $\geq 2.5 \mu \mathrm{IU} / \mathrm{mL})$.

The distribution of patients by gender showed a slight predominance of females over males (50.50\% vs $49.50 \%)$. Paruk et al (2016) found that $55.50 \%$ of the population diagnosed with type 1 diabetic thyroiditis were females versus $44.5 \%$ males with a sex ratio of 0.8016 . The same observations have been made by Umpierrez et al (2003) and Kakleas et al (2015) in longitudinal studies 7,17. Our results are in accordance with the European follow-up studies conclusion where thyroid autoimmunity can be associated with increased age, female gender, and long diabetes duration 17,18. Another interesting finding of the present study was that there was no significant association between increased TSH levels and BMI status in T1D patients, which is an agreement with certain studies 17,18.

In our patients' group, we have noticed a significant association between blood pressure (SBP or DBP) and hypertension with higher serum TSH levels. Our findings are in agreement with the previous study which demonstrates that thyroid hormone deficiency has serious effects on the cardiovascular system, and overt hypothyroidism leads to substantial alterations of traditional risk factors for cardiovascular disease including diastolic hypertension and increased systemic vascular resistance, decreased cardiac preload and impaired cardiac performance 19. Hypothyroidism prevalence was similar to that reported by Paruk et al ${ }^{16}$. However, the prevalence from other African countries including Uganda, Egypt, and Libya ranged between $4.2 \%$ and $7.3 \%$ which is lower than what we reported 20-22, meanwhile, is also known that hypothyroidism generates opposite cardiovascular changes and is associated with the increased risk for coronary atherosclerosis 12 . With regard to the findings of hyperthyroidism, the prevalence reported by Ghawil et al (2011) was $1 \%$ 22. Whereas our data showed that approximately $6.3 \%$ of our patients were affected.
In our study, there was no difference between HbA1c levels in both groups. These results are in accordance with reports from the literature 17,23 .

Our results showed that there is a significant increase in hsCRP levels among patients with higher TSH concentrations. Comparable findings regarding increased inflammatory markers such as hs-CRP were encountered in diabetic patients (type 1 or type 2 diabetes) with thyroid hormone abnormalities, represented by higher hs-CRP levels ${ }^{24}$.In addition, other studies in people with T1D have confirmed the relationship between elevated hs-CRP and atherosclerotic cardiovascular disease which reflects high grade atherogenic vascular inflammation process 25 .

We also identified that patients with increased TSH levels and AITD accompanying T1D had a worse lipid profile and their HDL-cholesterol level was significantly lower and triglyceride concentration was higher, these results concord with documented literature 26,27 .

An important observation of our study was the association of high serum TSH concentrations with increased urine albumin excretion in patients with T1D. Our findings echoes with the observations made by Das et al who established a similar relationship between increased prevalence of microalbuminuria and elevated TSH quartiles in British retrospective cross-sectional study 28.

Microalbuminuria is well documented in T1D and usually represents endothelial dysfunction by increasing the risk of atherosclerotic cardiovascular disease 14,29 . Therefore, it can be used as a marker of trans-capillary leakage of lipoproteins and systemic vascular damage may play role in accelerating atherosclerosis 30 .

In this study, the overall prevalence of Anti-TPO positivity was $16.31 \%$. These results are similar to those of the literature in particular Paruk's et al 18.1\% 16, and also Ghawil et al $16.5 \%{ }^{22}$. The prevalence is also similar to the 15\%-30\% figure reported in the literature for Western countries ${ }^{31}$. However, this rate is higher than the $7.3 \%$, and $9.5 \%$, reported in other African studies 20,21.

In the present study, the ability of higher tops quartiles versus $1^{\text {st }}$ quartile of lipid ratios (TC/HDL, LDL/HDL, and TG/HDL) in association with high versus low-atherosclerosis risk in type 1 diabetic patients was determined according to their TSH levels with the calculation of odds ratio (OR) and the corresponding 95\% confidence interval using logistic regression.

The association of TC/HDL and LDL/HDL with atherosclerosis risk at the two higher quartiles was stronger but non-significant, especially in the group of T1D with TSH $\geq 2.5 \mu \mathrm{IU} / \mathrm{mL}$. All the same, the $4^{\text {th }}$ and the $2^{\text {nd }}$ quartiles of TG/HDL and ratio were significantly higher in T1D patients with increased levels of TSH, indicating relatively increased dyslipidemia, as evidenced through LDL and HDL levels, and an increased cardiovascular risk mainly in these patients. The same conclusions have been reported in a study done by Shivakrishna et al. (2013) 32. Several case-control studies have also established an association between hypothyroidism and atherosclerosis 33,34. In their study of subjects with TSH levels of $4.0 \mathrm{mU} /$ liter or more, Tieche et al. reported a higher prevalence of coronary heart disease compared to controls with low TSH levels age-matched 35.

Undeniably, through lipid metabolism disorder, subclinical hypothyroidism contributes to vascular endothelial dysfunction. The results of a meta-analysis of 27 casecontrol studies revealed that elevated serum TSH levels were positively correlated with dyslipidemia and markers of 
cardiovascular risk 36. Most recent studies also indicate a close correlation between hypothyroidism, artery wall thickness, stiffness, endothelial dysfunction, and an increased risk of cardiovascular disease 37 .

In addition, the risk of developing atherosclerosis due to high TSH levels has been identified as related to the patient's gender. Furthermore, lipid ratio rates were significantly higher in men than in women. Thus, the results of the present study indicate that sex hormones may actively contribute to atherosclerosis disease development. Li et al. and Tognini et al. also found that the gender difference had considerable effects on the association between TSH levels and serum lipid levels, and their conclusions were in agreement with those of the present study 38,39 .

Several hypotheses explain the mechanism of the effects of sex hormones on lipid metabolism, testosterone and estradiol have different roles. The lipolytic enzymes LPL and HL remove TG from the bloodstream and lower serum chylomicron levels. Androgens play a stimulating role for HL activity, however, estrogens inhibit HL and LPL activities 40. Testosterone increases lipolysis by stimulating $\beta$-adrenergic activity, regulates the genes responsible for the catabolism of HDL, and increases the activity of hepatic lipase 41 . Estrogen, on the other hand, is capable of increasing cholesterol and LDL levels 42 .

In the current study, we investigated anti-TPO levels according to TSH concentrations. Elevated anti-TPO levels were associated with higher TSH values. Our results could be supported by both Whickham conclusions and Bromińska findings who reported that elevated serum TSH and TPO antibodies levels, separately or together are a risk factor for hypothyroidism ${ }^{43}$.

According to the Rotterdam study conducted by Hak et al., an atherogenic role for thyroid antibodies has been suggested since a higher incidence of atherosclerosis has been noted in hypothyroid anti-TPO positive patients 44 . Chronic inflammation in thyroid antibody-positive patients is one of the mechanisms behind this condition, ultimately leading to atherosclerosis 45,46 .

In this study, we have certain limitations that need to be considered. First, the lack of genetic analysis and the absence of control subjects were excluded because of cost constraints. Second, the temporal relationship between thyroid dysfunction and the presence of anti-TPO could be more assessed by other laboratory measurements such as free triiodothyronine (fT 3 and fT 4) concentrations and autoantibodies against thyroglobulin (anti-TG). Therefore, only TSH levels and anti-TPO were used to determine the patients' thyroid status. Despite these limitations, our conclusions allow us to comment on the employment of lipid ratios in the evaluation of atherosclerosis risk in patients with T1D as a vigorous method regardless of gender and TSH status. Likewise, data from the present investigation can be good support for further research due to the limited studies concerning atherosclerosis in type 1 diabetic patients with thyroid dysfunction.

In conclusion, this study's findings of borderline elevated levels of triglycerides and decreased HDL-cholesterol concentrations associated with higher serum TSH levels in patients with T1D make it reasonable to suggest an additional increase of cardiovascular risk for affected patients. AITD may especially affect cardiovascular morbidity and mortality in diabetic patients and deteriorated lipoprotein levels represent potentially modifiable risk factors. Therefore, an early screening of potential thyroid dysfunction and associated dyslipidemia is essential for effective prevention of premature heart failure.

\section{ACKNOWLEDGEMENTS}

The authors thank all the members of the Endocrinology Department of the University Hospital Center for their support to this study. I also want to express my deepest appreciation to Pr. Hadj Habib who assisted me during data collection.

\section{CONFLICT OF INTEREST}

The authors declare no conflicts of interest.

\section{REFERENCES}

1. International Diabetes Federation. IDF Diabetes Atlas. $9^{\text {th }}$ ed. Brussels: International Diabetes Federation; 2019. P. 34-55.

2. Oh KY, Kim YH, Yang EM, Kim CJ. Frequency of diabetes and thyroid autoantibodies in patients with type 1 diabetes and their siblings. Chonnam Med J, 2016; 52(2):136-140.

3. Touhami M, Zennaki A, Bouchetara A, Naceur M, Aoui A, Gharnouti M, et al. Epidemiological Evolution of Type 1 Diabetes in Children: Data from the Register of the Department of Oran, Algeria, 1973-2017. Revue d'epidemiologie et de sante publique, 2019; 67(6):369-374.

4. Dittmar M, Kahaly GJ. Genetics of the autoimmune polyglandular syndrome type 3 variant. Thyroid, 2010; 20(7):737-743.

5. Barker JM. Type 1 diabetes-associated autoimmunity: natural history, genetic associations, and screening. J Clin Endocrinol Metab, 2006; 91(4):1210-1217.

6. Tomer Y, Menconi F. Type 1 diabetes and autoimmune thyroiditis: the genetic connection. Thyroid, 2009; 19(2):99102.

7. Umpierrez GE, Latif KA, Murphy MB, Lambeth HC, Stentz F, Bush A, et al. Thyroid dysfunction in patients with type 1 diabetes: a longitudinal study. Diabetes Care, 2003; 26(4):1181-1185

8. Shun CB, Donaghue KC, Phelan H, Twigg SM, Craig ME. Thyroid autoimmunity in type 1 diabetes: systematic review and metaanalysis. Diabet Med, 2014; 31(2):126-135

9. Witek P, Witek J, Pańkowska E. Type 1 diabetes-associated autoimmune diseases: screening, diagnostics principles and management. Dev Period Med, 2012; 31(2):23-34.

10. American Diabetes Association and others. Sec.11.Children and adolescents. Diabetes Care, 2015; 38(Suppl 1):S70-76.

11. Cappola AR, Ladenson PW. Hypothyroidism and atherosclerosis. J Clin Endocrinol Metab, 2003; 88(6): 2438-2444.

12. Ichiki T. Thyroid hormone and atherosclerosis. Vascul Pharmacol, 2010; 52(3-4):151-156.

13. Lan N S, Fegan PG, Yeap BB, Bell DA, Watts GF. Dyslipidaemia in adults with type 1 diabetes-when to treat?. Diabetes/metabolism research and reviews, 2019; 35(1):e3090.

14. De Ferranti SD, de Boer IH, Fonseca V, Fox CS, Golden SH, Lavie CJ, et al. Type 1 diabetes mellitus and cardiovascular disease: a scientific statement from the American Heart Association and American Diabetes Association. Circulation, 2014; 130(13):1110-1130.

15. World Health Organization and others. Definition and diagnosis of diabetes mellitus and intermediate hyperglycaemia: report of a WHO/IDF consultation. Geneva:WHO; 2006.

16. Paruk IM., Ganie Y, Maharaj S, Pirie FJ, Naidoo VG, Nkwanyana $\mathrm{NM}$, et al. High prevalence of antithyroid peroxidase and antiparietal cell antibodies among patients with type 1 diabetes mellitus attending a tertiary diabetes centre in South Africa. Postgraduate medical journal, 2017; 93(1100):338-343.

17. Kakleas K, Paschali E, Kefalas N, Fotinou A, Kanariou M, Karayianni C, et al. Factors for thyroid autoimmunity in children and adolescents with type 1 diabetes mellitus. Upsala Journal of Medical Sciences, 2009; 114(4):214-220.

18. ORZAN A, NOVAC C, Mihaiela MIHU, TIRGOVISTE CI, BALGRADEAN M. Type 1 diabetes and thyroid autoimmunity in children. Maedica, 2016; 11(4):308.

19. Biondi B, Cooper DS. The clinical significance of subclinical thyroid dysfunction. Endocrine Reviews, 2008; 29(1):76-131.

20. Muhame RM, Mworozi EA, McAssey K, Lubega I. Thyroid autoimmunity and function among Ugandan children and adolescents with type-1 diabetes mellitus. Pan Afr Med J, 2014; 19:137. 
21. Metwalley KA, El-Saied ARAH. Thyroid abnormalities in Egyptian children and adolescents with type 1 diabetes mellitus: a single center study from Upper Egypt. Indian J Endocrinol Metab, 2014; 18(5):637-641.

22. Ghawil M, Tonutti E, Abusrewil S, Visentini D, Hadeed I, Miotti V, et al. Autoimmune thyroid disease in Libyan children and young adults with type 1 diabetes mellitus. European journal of pediatrics, 2011; 170(8):983-987.

23. Grzelka A, Araszkiewicz A, Uruska A, Zozulińska-Ziółkiewicz D. Prevalence of anti-thyroid peroxidase in adults with type 1 diabetes participating in Poznań Prospective Study. Advances in Clinical and Experimental Medicine, 2015; 24(1):79-84.

24. Neto AM, Parisi MCR, Alegre SM, Pavin EJ, Tambascia MA Zantut-Wittmann DE. Relation of thyroid hormone abnormalities with subclinical inflammatory activity in patients with type 1 and type 2 diabetes mellitus. Endocrine, 2016; 51(1):63-71.

25. Jellinger PS, Handelsman Y, Rosenblit PD, Bloomgarden ZT, Fonseca VA, Garber AJ, et al. American Association of Clinical Endocrinologists and American College of Endocrinology guidelines for management of dyslipidemia and prevention of cardiovascular disease. Endocrine practice, 2017; 23(Suppl 2):1-87.

26. Korzeniowska K, Ramotowska A, Szypowska A, Szadkowska A, Fendler W, Kalina-Faska B, et al. How does autoimmune thyroiditis in children with type 1 diabetes mellitus influence glycemic control, lipid profile and thyroid volume?. Journal of Pediatric Endocrinology and Metabolism, 2015; 28(3-4):275278.

27. Asvold BO, Vatten LJ, Nilsen TI, Bjøro T. The association between TSH within the reference range and serum lipid concentrations in a population-based study. The HUNT Study. Eur J Endocrinol, 2007; 156(2):181-186.

28. Das G, Taylor PN, Abusahmin H, Ali A, Tennant BP, Geen J, et al. Relationship between serum thyrotropin and urine albumin excretion in euthyroid subjects with diabetes. Annals of clinical biochemistry, 2019; 56(1):155-162.

29. Bertoluci MC, Cé GV, da Silva AM, Wainstein MV, Boff W, Puñales M. Endothelial dysfunction as a predictor of cardiovascular disease in type 1 diabetes. World journal of diabetes, 2015; 6(5):679.

30. Jensen JS, Feldt-Rasmussen B, Borch-Johnsen K, Jensen KS, Nordestgaard BG. Increased transvascular lipoprotein transport in diabetes: association with albuminuria and systolic hypertension. The Journal of clinical endocrinology and metabolism, 2005; 90(8):4441-4445.

31. Van den Driessche A, Eenkhoorn V, Van Gaal L, De Block C. Type 1 diabetes and autoimmune polyglandular syndrome: a clinical review. Neth J Med, 2009; 67(11):376-387.

32. Shivakrishna G, Reddy S, Varikasuvu ARB, Sachan A, Suresh V, Lalitha D, et al. Evaluation of Dyslipidemia, Lipid Ratios and Atherogenic Index as Cardiovascular Risk Factors in Overt and Subclinical Hypothyroid Patients. National Journal of Laboratory Medicine, 2013; 2(3):11-15.
33. Chaker L, Baumgartner C, Den Elzen WP, Collet TH, Ikram MA, Blum MR, et al. Thyroid function within the reference range and the risk of stroke: an individual participant data analysis. J Clin Endocrinol Metab, 2016; 101(11):4270-4282.

34. Gussekloo J, Van Exel E, de Craen AJ, Meinders AE, Frölich M, Westendorp RG. Thyroid status, disability and cognitive function, and survival in old age. JAMA, 2004; 292(21):25912599.

35. Tieche M, Lupi GA, Gutzwiller F, Grob PJ, Studer H, Burgi H Borderline low thyroid function and thyroid autoimmunity. Risk factors for coronary heart disease?. Br Heart J, 1981; 46(2):202-206.

36. Yao K, Zhao T, Zeng L, Yang J, Liu Y, He Q, et al. Non-invasive markers of cardiovascular risk in patients with subclinical hypothyroidism: A systematic review and meta-analysis of 27 case control studies. Sci Rep, 2018; 8(1):1-11.

37. Lai Y, Wang J, Jiang F, Wang B, Chen Y, Li M, et al. The relationship between serum thyrotropin and components of metabolic syndrome. Endocr J, 2010; 58:23-30.

38. Li X, Meng Z, Tan J, Liu M, Jia Q, Zhang G, et al. Gender impact on the correlation between thyroid function and serum lipids in patients with differentiated thyroid cancer. Exp Ther Med, 2016; 12(5):2873-2880.

39. Tognini S, Polini A, Pasqualetti G, Ursino S, Caraccio N, Ferdeghini M, et al. Age and gender substantially influence the relationship between thyroid status and the lipoprotein profile: Results from a large cross-sectional study. Thyroid, 2012; 22(11):1096-1103.

40. Agledahl I, Skjaerpe PA, Hansen JB, Svartberg J. Low serum testosterone in men is inversely associated with non-fasting serum triglycerides: The Troms $\varnothing$ study. Nutr Metab Cardiovasc Dis, 2008; 18(4):256-262.

41. Haffner SM, Valdez RA. Endogenous sex hormones: Impact on lipids, lipoproteins and insulin. Am J Med, 1995; 98(1):S40-S47.

42. Pellegrini M, Pallottini V, Marin R, Marino M. Role of the sex hormone estrogen in the prevention of lipid disorder. Curr Med Chem, 2014; 21(24):2734-2742.

43. Hoogendoorn EH, Hermus AR, De Vegt F, Ross HA, Verbeek AL, Kiemeney LA, et al. Thyroid function and prevalence of antithyroperoxidase antibodies in a population with borderline sufficient iodine intake: influences of age and sex. Clin Chem, 2006; 52(1):104-111.

44. Hak AE, Pols HA, Visser TJ, Drexhage HA, Hofman A, Witteman JC. Subclinical hypothyroidism is an independent risk factor for atherosclerosis and myocardial infection in elderly women: the Rotterdam Study. Ann Intern Med, 2000; 132(4):270-278.

45. Raterman HG, Voskuyl AE, Simsek S, Schreurs MWJ, Van Hoogstraten IM, Peters MJ, et al. Increased progression of carotid intima media thickness in thyroid peroxidase antibodies-positive rheumatoid arthritis patients. Eur J Endocrinol, 2013; 169(6):751-757.

46. İşüven P, Gündüz Y, Kılıç M. Effects of Thyroid Autoimmunity on Early Atherosclerosis in Euthyroid Girls with Hashimoto's Thyroiditis. J Clin Res Pediatr Endocrinol, 2016; 8(2):150-156. 